\title{
Skin regeneration in deep second-degree scald injuries either by infusion pumping or topical application of recombinant human erythropoietin gel
}

This article was published in the following Dove Press journal:

Drug Design, Development and Therapy

II May 2015

Number of times this article has been viewed

\author{
Priya Giri' \\ Sabine Ebert ${ }^{1}$ \\ Ulf-Dietrich Braumann² \\ Mathias Kremer ${ }^{3}$ \\ Shibashish Giri' \\ Hans-Günther Machens ${ }^{4}$ \\ Augustinus Bader' \\ 'Department of Cell Techniques and \\ Applied Stem Cell Biology, Center \\ for Biotechnology and Biomedicine \\ (BBZ), Faculty of Medicine, \\ University of Leipzig, Leipzig, \\ Germany; ${ }^{2}$ Interdisciplinary Center \\ for Bioinformatics (IZBI), University \\ of Leipzig, Leipzig, Germany; \\ ${ }^{3}$ Department of Plastic and Hand \\ Surgery, University of Lübeck, Lübeck, \\ Germany; ${ }^{4}$ Department of Plastic \\ and Hand Surgery, Technical University \\ of Munich, Munich, Germany
}

\begin{abstract}
Large doses of recombinant growth factors formulated in solution form directly injected into the body is usual clinical practice in treating second-degree scald injuries, with promising results, but this approach creates side effects; furthermore, it may not allow appropriate levels of the factor to be sensed by the target injured tissue/organ in the specific time frame, owing to complications arising from regeneration. In this research, two delivery methods (infusion pumping and local topical application) were applied to deliver recombinant human erythropoietin (rHuEPO) for skin regeneration. First, rHuEPO was given in deep second-degree scald injury sites in mice by infusion pump. Vascularization was remarkably higher in the rHuEPO pumping group than in controls. Second, local topical application of rHuEPO gel was given in deep second-degree scald injury sites in rats. Histological analysis showed that epithelialization rate was significantly higher in the rHuEPO gel-treated group than in controls. Immunohistochemical studies showed that the rHuEPO gel-treated group showed remarkably higher expression of skin regeneration makers than the control group. An accurate method for visualization and quantification of blood vessel networks in target areas has still not been developed up to this point, because of technical difficulties in detecting such thin blood vessels. A method which utilizes a series of steps to enhance the image, removes noise from image background, and tracks the vessels edges for vessel segmentation and quantification has been used in this study. Using image analysis methods, we were able to detect the microvascular networks of newly formed blood vessels (less than $500 \mu \mathrm{m}$ thickness), which participate in the healing process, providing not only nutrition and oxygen to grow tissues but also necessary growth factors to grow tissue cells for complete skin regeneration. The rHuEPO-treated group showed higher expression of stem cell markers (CD 31, CD 90, CD 71, and nestin), which actively contribute to in-wound-healing processes for new hair follicle generation as well as skin regeneration. Collectively, both rHuEPO group pumping into the systemic circulation system, and injection into the local injury area, prompted mice and rats to form new blood vessel networks in scald injury sites, which significantly participate in the scald healing process. These results may lead to the development of novel treatments for scald wounds.
\end{abstract}

Keywords: re-epithelialization, scald wound, skin regeneration, neovascularization, vascularization, segmentation

\section{Introduction}

Scald injuries ranked in the top 15 leading causes of the burden of disease worldwide, ${ }^{1}$ and annual deaths from scalds injuries are approximately 300,000 worldwide. ${ }^{2}$ Scald injuries are also among the most expensive traumatic injuries because of the long recovery period. ${ }^{3}$ Annually, approximately six million patients seek medical treatment for scalds worldwide, but the majority are often treated in outpatient clinics. ${ }^{4}$ 
Scald injuries can be fatal for all age groups; however, elderly and very young people are at a higher risk. ${ }^{5}$ Even small scalds (defined as having a mean $7 \%$ total body surface area) may lead to high mortality $(22 \%){ }^{6}$ According to a US Centers for Disease Control and Prevention (CDC) report, the fourth leading cause of unintentional injury mortality among people aged 65 years or older is because of scald-related injuries. ${ }^{7}$ Therefore, treating scalds has now became a substantial challenge in clinical practice.

Neovascularization is the crucial step for regeneration of skin. Topical application of antibiotics has been used to treat scald areas in patients ${ }^{8}$ for protection from microorganisms, but this treatment does not promote skin regeneration. Recombinant human erythropoietin (rHuEPO) stimulates revascularization, angiogenesis, and wound healing in genetically diabetic animal models as well as humans. ${ }^{9-14}$ Previously, we reported the first successful clinical case of topical application of rHuEPO in acute and chronic wounds in humans. ${ }^{9}$ Furthermore, we proved that granulocyte colony-stimulating factor played an active role in boosting autologous mesenchymal stem cell recruitment and that rHuEPO increased angiogenesis in a stem-cell-based, tissueengineered tracheal replacement in a 12 -year-old boy. ${ }^{10}$

Angiogenesis and neovascularization, are mediated in large part by cytokines and growth factors, ${ }^{15,16}$ which are key components involved in the healing of scald injuries. ${ }^{17}$ Scald injuries often lose dermal vascular blood vessel network flow, and these vessels are then unable to bring oxygen and nutrients to injured tissues for regeneration. Angiogenesis and neovascularization not only allow the transport of oxygen and nutrients to injured tissues, but also improve the intake of inflammatory cells, stimulating the scalded areas to allow better skin regeneration and recovery.

Evidence accumulating from both in vitro and in vivo models has indicated that $\mathrm{rHuEPO}$ has an outstanding role in stimulating angiogenesis and generation of new blood vessels from pre-existing vessels. ${ }^{18-20}$ Recently, it was reported that $\mathrm{rHuEPO}$ quickens angiogenesis by the upregulation of systemic levels of hepatocyte growth factor and fibroblast growth factor, and the local expression of vascular endothelial growth factor and insulin-like growth factor in a porcine myocardial infarction model. ${ }^{21}$ In the current study, two methods (infusion pumping and local topical application) are applied to deliver rHuEPO in two animal models (mouse and rat). We aim to evaluate the role of rHuEPO in neovascularization, with special reference to quantification of newly formed blood vessels in the deep second-degree skin scald injuries in a mouse model using the infusion pumping method.
This infusion pumping method is designed to mimic the in vivo circulation process. Secondly, similar to our previous treatment of scalds in human acute and chronic wounds, ${ }^{9}$ local topical application of rHuEPO gel was applied to scald injury sites in rats and evaluated by epithelialization rate and the major stem cell-related markers (CD 90, CD 71, CD 31, and nestin) for neovascularization, which is a marker for skin regeneration.

Developing and accurate ex vivo technique for visualizing and accurate quantification of microvasculature of blood vessels is still a challenging problem in many biological studies, ${ }^{22}$ including the clinical observation of different angiogenesis and neovascularization processes. In the present study, the presence of morphological changes in angiogenesis and neovascularization in mice wounded by scalds was focused on using an ex vivo image analysis assessment method. A reliable ex vivo method for segmentation of blood vessels in scald models is still difficult to find. Existing techniques such as fluorescence, and confocal and electron microscopy have been used conventionally for visualization and accurate quantification of microvasculature of blood vessels. However, these techniques are laborious, time consuming, and expensive. Previously, we introduced a technique to detect and to quantify fasciocutaneous blood vessels in small laboratory animals ex vivo. ${ }^{23}$ Previously, we also reported visualization of fasciocutaneous blood vessels by digital mammography and analyzed images for vessel length and vessel surface area as standardized units by computer program. ${ }^{24}$ In the current research, partially segmented data with approximately identified vessel centerlines were used to comprehensively visualize and accurately quantify the microvasculature of blood vessels in a scalded mouse. In addition, we quantified the vascularization levels of the better examples of skin regeneration, describing a new, easy, and quick ex vivo technique that allows visualization and quantification of area and length of blood vessel networks in scalded mice, using both the rHuEPO group and the untreated group as models.

\section{Methods}

\section{Scalded mouse model}

The experiments were performed in adherence to the Declaration of Helsinki and the Guide for the Care and Use of Laboratory Animals, and were approved by the Ethics Committee of the University of Lübeck, Lübeck, Germany. Thirty-five mixed female BALB/c and C57BL/6 mice (aged 6-8 weeks, each weighing 20-25 g, and all sourced from Takomi, Copenhagen, Denmark) received a second-degree 
scald wound by previously reported methods. ${ }^{25}$ Animals were anesthetized with a mix of ketamine $(10 \mathrm{mg} / \mathrm{kg})$ and xylazine $(2.4 \mathrm{mg} / \mathrm{kg})$ via intraperitoneal injection. Under general anesthesia, hair on the back was shaved and the skin was washed with sterile water. Mice were inserted headfirst into a scald template. The dorsum was centered by a $2 \times 3 \mathrm{~cm}$ window and then immersed in $80^{\circ} \mathrm{C}$ water for 6 seconds. By this method, we produced a second-degree scald wound. A $2 \times 3 \mathrm{~cm}$ deep dermal scald injury defect was created in the backs of the animals. One group (group A) was treated with rHuEPO; the control group (group B) received only saline water (vehicle) without $\mathrm{rHuEPO}$. After 7 days, the scalded tissue was harvested, and the regeneration rate was measured. Figure 1A shows our deep dermal scald injury regeneration model (the scale bar represents $1 \mathrm{~cm}$ ). Scalded mice were immediately administered with $1 \mathrm{~mL} / \mathrm{kg}$

A

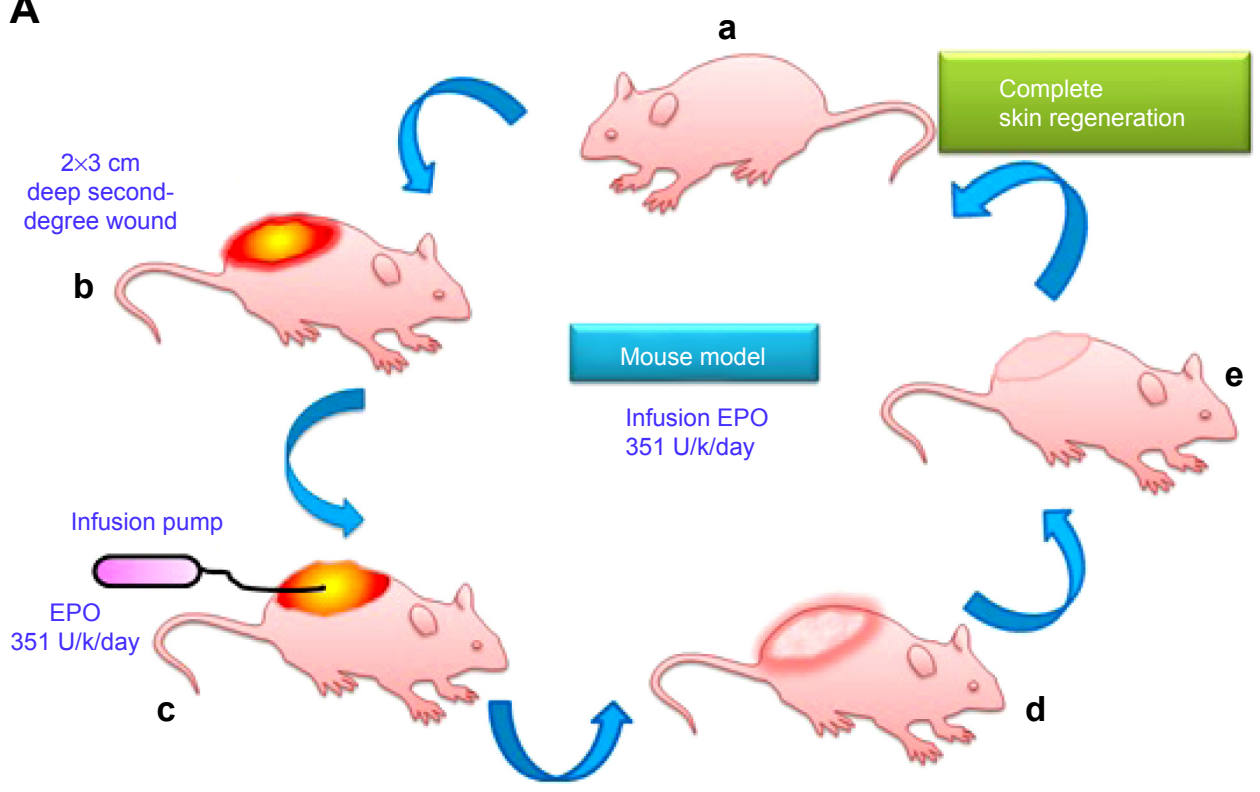

B

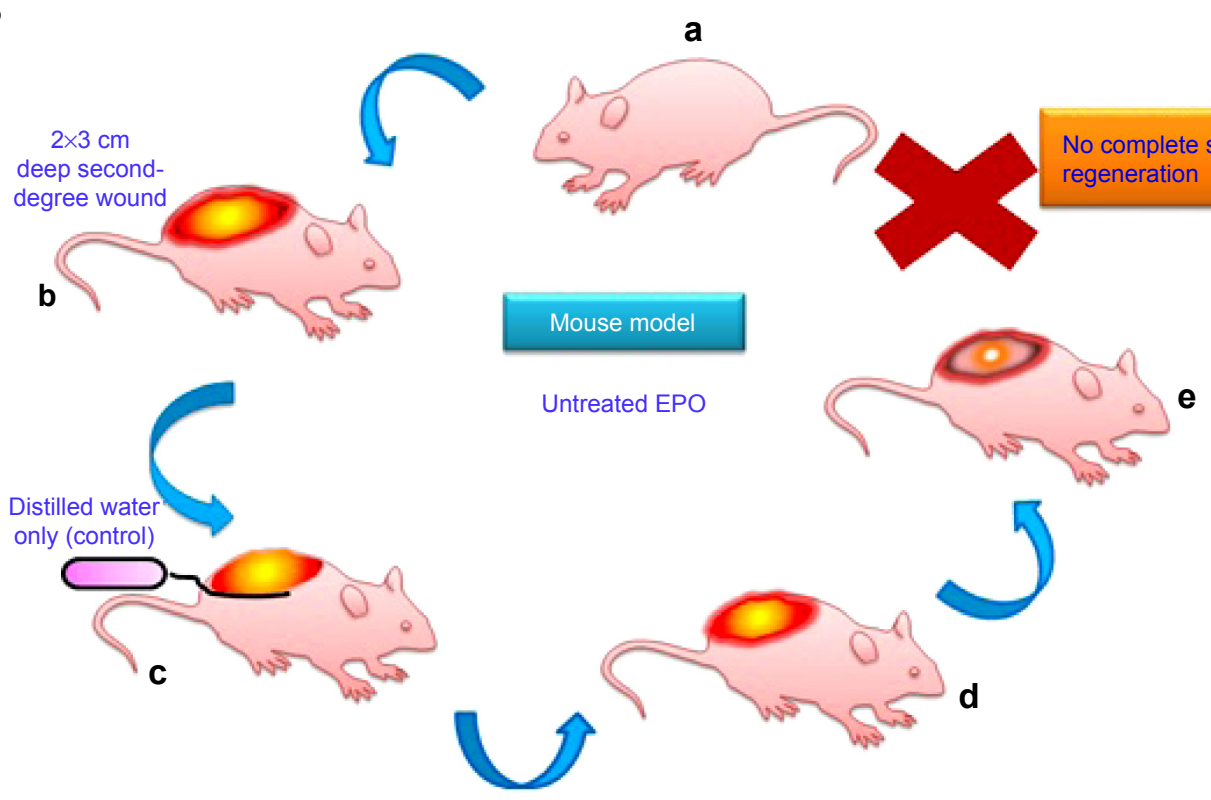

Figure I Diagrammatic representation of EPO treated and untreated mouse model for skin regeneration.

Notes: Scald mouse model (ages, 6-8 weeks; weights, 20-25 g), EPO treated (A, a-e). The dorsum was centered by a $2 \times 3 \mathrm{~cm}$ window and then immersed in $80^{\circ} \mathrm{C}$ water for 6 seconds to produce a second-degree scald wound. A $2 \times 3 \mathrm{~cm}$ deep dermal scald injury defect was created in the backs of the animals. This group was treated with rHuEPO ( $352 \mathrm{U} / \mathrm{kg} /$ day) by infusion pump, an attempt to mimic the in vivo circulation process. Scald mouse model (ages, 6-8 weeks; weights, 20-25 g), untreated EPO (B, a-e). The dorsum was centered by a $2 \times 3 \mathrm{~cm}$ window and then immersed in $80^{\circ} \mathrm{C}$ water for 6 seconds to produce a second-degree scald wound. A $2 \times 3 \mathrm{~cm}$ deep dermal scald injury defect was created in the backs of the animals. This group was treated with distilled water (control model) only by infusion pump.

Abbreviations: EPO, erythropoietin; rHuEPO, recombinant human erythropoietin. 
saline Ringer's solution. Animals were then housed one per cage, and the wound was appropriately covered with a hydrocolloid dressing to prevent animals from grooming or licking.

After thermal injury, animals were randomized into two groups. The animals were then implanted with subcutaneous pumps (ALZET ${ }^{\circledR}$ osmotic pumps, Model 1002, delivery rate $0.25 \mu \mathrm{L} /$ hour [DURECT Corporation, Cupertino, CA, USA]), which continuously released $\mathrm{rHuEPO}$ at a rate of $352 \mathrm{U} / \mathrm{kg} /$ day or its vehicle (distilled water). The filled pump was implanted via a dermal incision on the back of the animal, slightly posterior to the scapula. The wound was closed with sutures. The dose and the type of rHuEPO were chosen in agreement with previous experimental data in mice showing that the hematopoietic factor improves wound healing in C57BL/6 mice. ${ }^{25}$ Postscald sedation and analgesia were provided for the first 7 days with novaminsulfon $(1,000 \mathrm{mg} / \mathrm{L}$ added to the drinking water). During the experiments, the animals were housed one per cage, under controlled environmental conditions (12-hour light/dark cycle, temperature approximately $23^{\circ} \mathrm{C}$ ), and provided with standard laboratory food and water ad libitum. At day 7, 14 animals were sacrificed with an overdose of anesthetics, and regenerated tissue was obtained for further analyses. The skin was removed from the back in its entirety for angiography, or wound areas were excised and partially frozen in liquid nitrogen or kept in formalin for other studies.

\section{Blood vessel visualization using image analysis}

Skin from the back of the mouse was removed and placed immediately over a transilluminator (model LP 5000K; Hama GmbH, Monheim, Germany). Digital pictures were captured in tagged image file (Olympus camera model C-5060, 5.1 megapixels; Olympus Corporation, Tokyo, Japan) and stored for further digital analysis using the computer algebra system Mathematica, version 8 (Wolfram Research, Inc., Champaign, IL, USA), which offers a variety of image processing functions. Our method utilizes many steps (Figure 2) to enhance the image, remove noise from background of the image, and track the edges of the vessels. In attempting to locate clear views of blood vessels for the quantitative analysis, we have gone through certain images using precise steps such as smoothing, thresholding, skeletonizing, and distance transforming, etc. Regions of interest, as shown in the Figure $3 \mathrm{C}(\mathrm{b})$ and $\mathrm{D}(\mathrm{b})$ were selected from the sample image as shown in the Figure $3 \mathrm{C}(\mathrm{a})$ and $\mathrm{D}(\mathrm{a})$, and were then converted into grayscale as shown in Figure $3 \mathrm{C}(\mathrm{c})$ and $\mathrm{D}(\mathrm{c})$. A high-pass filter ${ }^{26}$ was used to remove inhomogeneous background as show in the Figure $3 \mathrm{C}(\mathrm{d})$ and $\mathrm{D}(\mathrm{d})$. An anisotropic diffusion filter ${ }^{27}$ was used for smoothing along the blood vessels while preserving their edges, as shown in the Figure $3 \mathrm{C}(\mathrm{e})$ and $\mathrm{D}(\mathrm{e})$. Global contrast enhancement was performed before global threshold was applied for binarization, as shown in Figure $3 \mathrm{C}(\mathrm{f}), \mathrm{D}(\mathrm{f}), \mathrm{C}(\mathrm{g})$, and $\mathrm{D}(\mathrm{g})$. We removed the minimum size of unwanted segments as shown in the Figure $3 \mathrm{C}(\mathrm{h})$ and $\mathrm{D}(\mathrm{h})$. Further, images were skeletonized as show in the Figure $3 \mathrm{C}(\mathrm{i})$ and $\mathrm{D}(\mathrm{i})$, and were then pruned to remove unwanted segments as shown in the Figure $3 \mathrm{C}(\mathrm{j})$ and $\mathrm{D}(\mathrm{j})$. Thickness measurements were made based on skeletonization ${ }^{28}$ of the blood vessels, while vessel width was computed using Euclidean distance transformation ${ }^{29}$ was applied on the vessel branches, as show in the Figure $3 \mathrm{C}(\mathrm{k})$ and $\mathrm{D}(\mathrm{k})$.

\section{The rat model of tangential excision}

Female rats (Charles Rivers CD-1 mice, 6-8 weeks of age) weighing 200-250 g were anesthetized with a mix of ketamine $(10 \mathrm{mg} / \mathrm{kg})$ and xylazine $(2.4 \mathrm{mg} / \mathrm{kg})$ via intraperitoneal injection. Under general anesthesia, hair on the back was shaved, and residual hair was removed with depilatory cream $\left(\right.$ Veet $^{\circledR}$; Reckitt Benckiser, Slough, UK). The skin was then washed with sterile water. With a dermatome (Aesculap Inc., Center Valley, PA, USA), each animal was given four dorsolateral skin defects $(1 \times 1.5 \mathrm{~cm})$ via tangential excision of a $0.2 \mathrm{~mm}$ section of skin. After thermal injury, the rats were randomly divided into two experimental groups of EPO treated $(n=11)$ and several control groups with no EPO given $(n=3)$. In the first experimental group, the rats were treated with $\mathrm{rHuEPO}$ hydrogel over the dermal wound bed every second day (150 IU $\mathrm{rHuEPO} / \mathrm{g}$, dose of $1 \mathrm{~g} / \mathrm{cm}^{2}$, preparation in Institute for Pharmaceutical Technology, Technical University, Braunschweig). In the second group, the control group, animals with thermal injuries received a vehicle preparation only (conventional hydrogel as placebo). The rHuEPO hydrogel 1 was prepared by mixing $150 \mathrm{IU} / \mathrm{mL}$ erythropoietin (EPO) in purified water for injection ( $\mathrm{pH} 7.8$ ) with $0.5 \%$ hydroxyethyl cellulose (Arseus, Waregram, Belgium) and 2.4\% glycerol free from water (Thermo Fisher Scientific, Waltham, MA, USA). Animals of one control group with thermal injuries generally received no treatment. Rats without injuries with the same gel application as the rHuEPO and control experimental groups described (with or without rHuEPO, respectively) and healthy rats without any treatment served as further controls. The dose and the type of rHuEPO were 


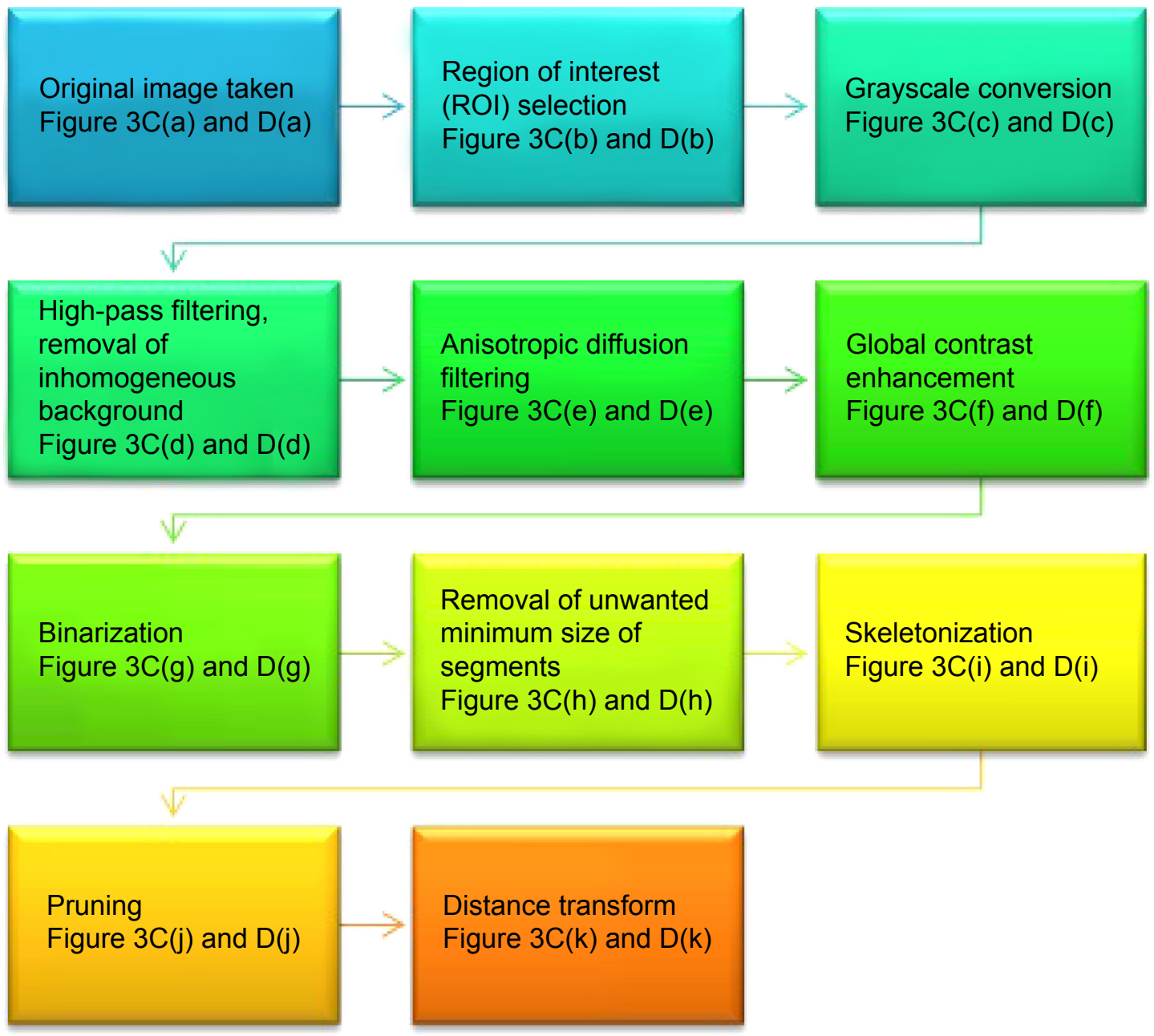

Figure 2 Flow chart of image analysis methods.

Notes: First, a series of steps to enhance the image, remove noise from background of the image, and track the edges of the vessels for blood vessel segmentation were applied. Quantification was also applied. Second, in attempting to locate a clear view of blood vessels for the quantitative analysis, we have followed a number of steps including smoothing, thresholding, skeletonizing, and distance transformation.

chosen according to a previous study in mice and rats showing the non-hematopoietic effect of rHuEPO in scald wound healing, ${ }^{25}$ with modification.

Animals were housed one per cage, and the wound was appropriately covered with a hydrocolloid dressing (Varihesive ${ }^{\circledR}$ E) to prevent animals from grooming or licking. The dressings were changed every second day in carbon dioxide-narcosis conditions and hydrogel, with or without $\mathrm{rHuEPO}$, was reapplied. Postoperative sedation and analgesia were provided with novaminsulfon $(1,000 \mathrm{mg} / \mathrm{L}$ added to the drinking water). During the experiments, the animals were held under controlled environmental conditions (12-hour light/dark cycle, temperature approximately $23^{\circ} \mathrm{C}$ ), and provided with standard laboratory food and water ad libitum. At days 4 and 7 , a defined quantity of rats in the experimental groups $(n=8)$ were sacrificed with an overdose of xylazine, and analyses for re-epithelialization levels were performed. At day 7, specimens from the remaining animals in the experimental groups were taken from the wound area, which was excised transdermally by scalpel. The rat skin tissue specimen were removed, immediately embedded in TissueTec (Leica Microsystems, Wetzlar, Germany) and frozen in liquid nitrogen for further histological studies.

\section{Quantification of the re-epithelialization levels}

In order to quantify re-epithelialization levels, digital pictures were obtained on days 0,4 , and 7 in tagged image file (TIF) format (Olympus camera, model C-5060, 5.1 Megapixels; Olympus Corporation). Re-epithelialization levels were measured using ImageJ in a blinded manner. The analysis software used in the present study can be downloaded free at http://rsb.info.nih.gov/ij. Results were expressed as a 
A
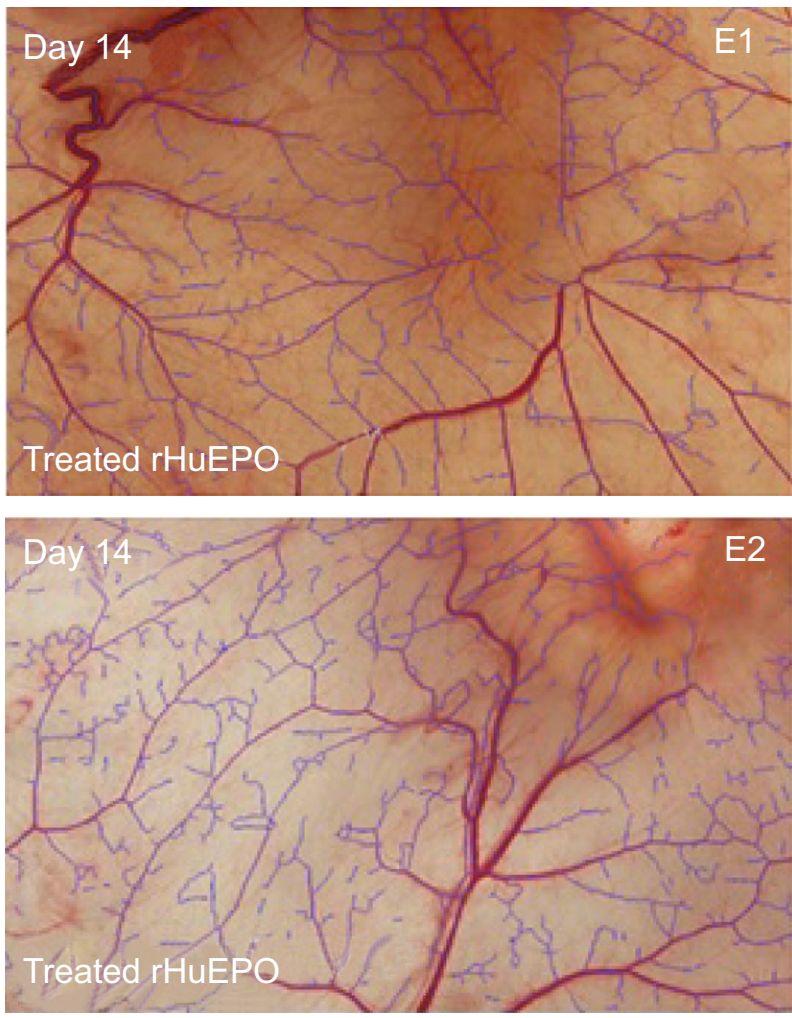

B
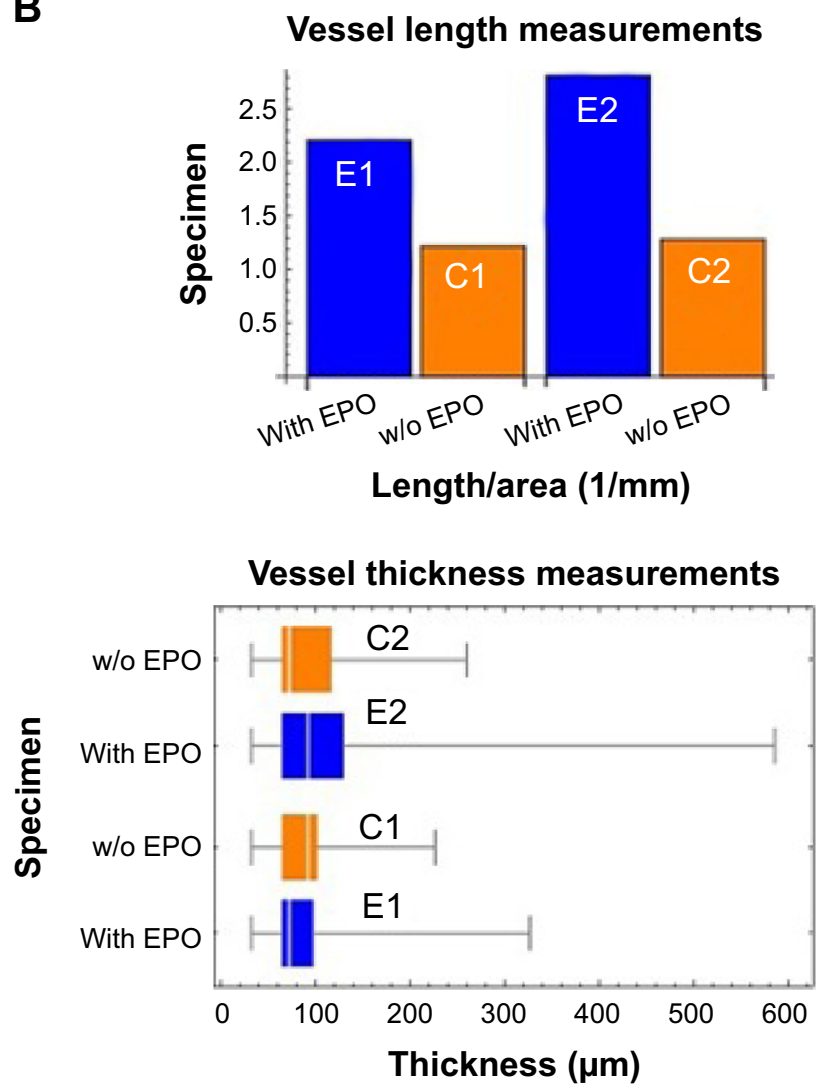
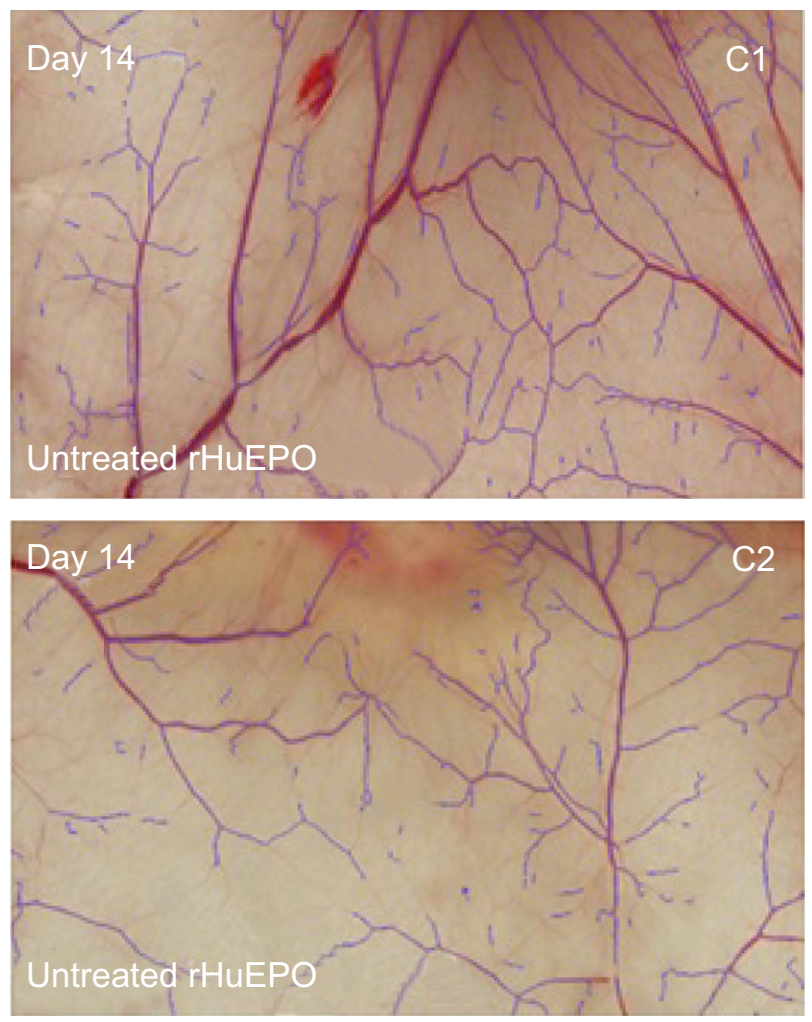

Average vessel length measurements

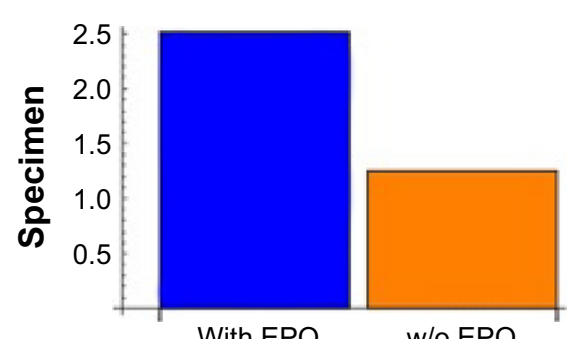

With EPO W/O EPO

Length/area $(1 / \mathrm{mm})$



Figure 3 (Continued) 
C
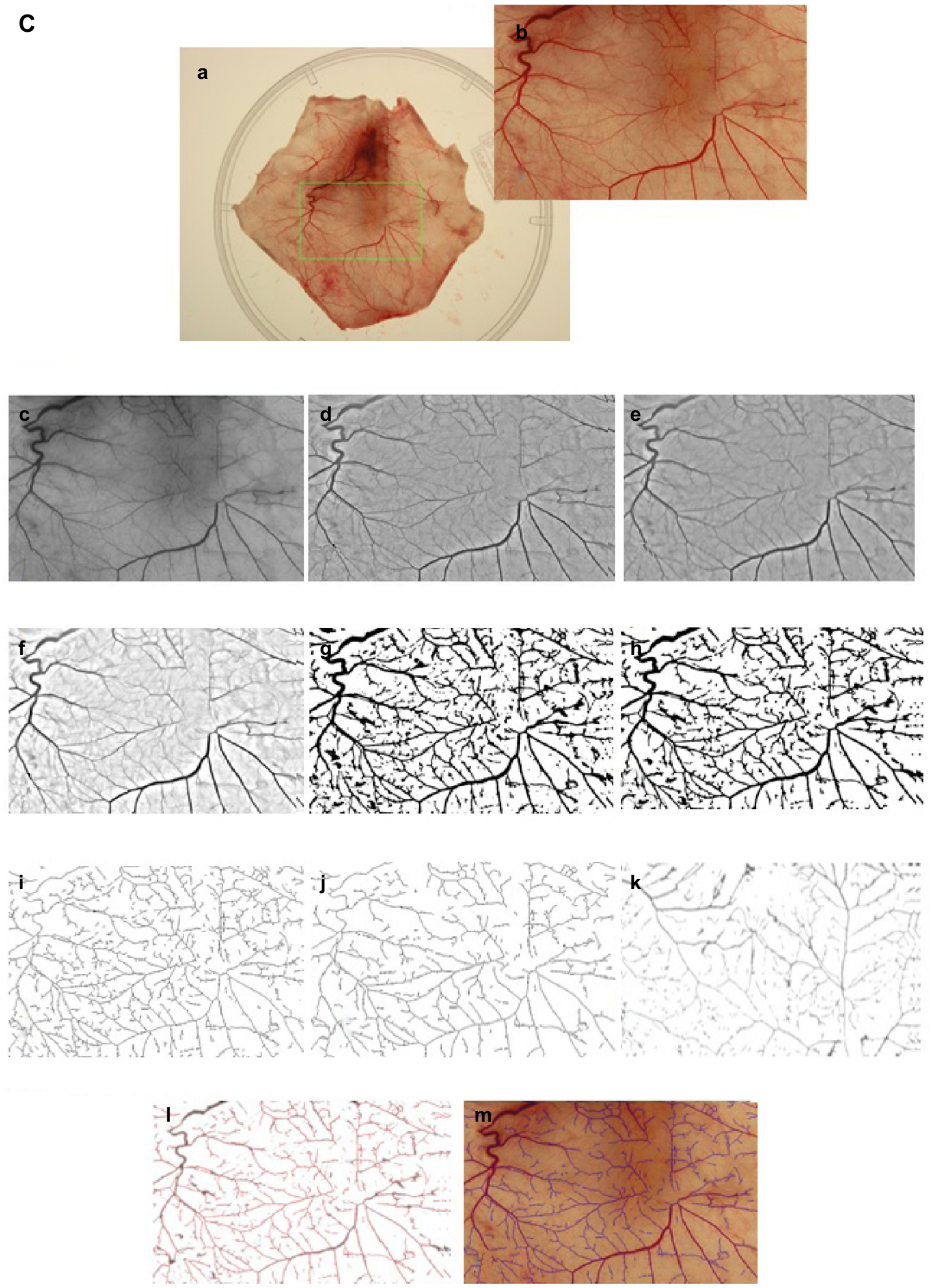

Figure 3 (Continued) 
D
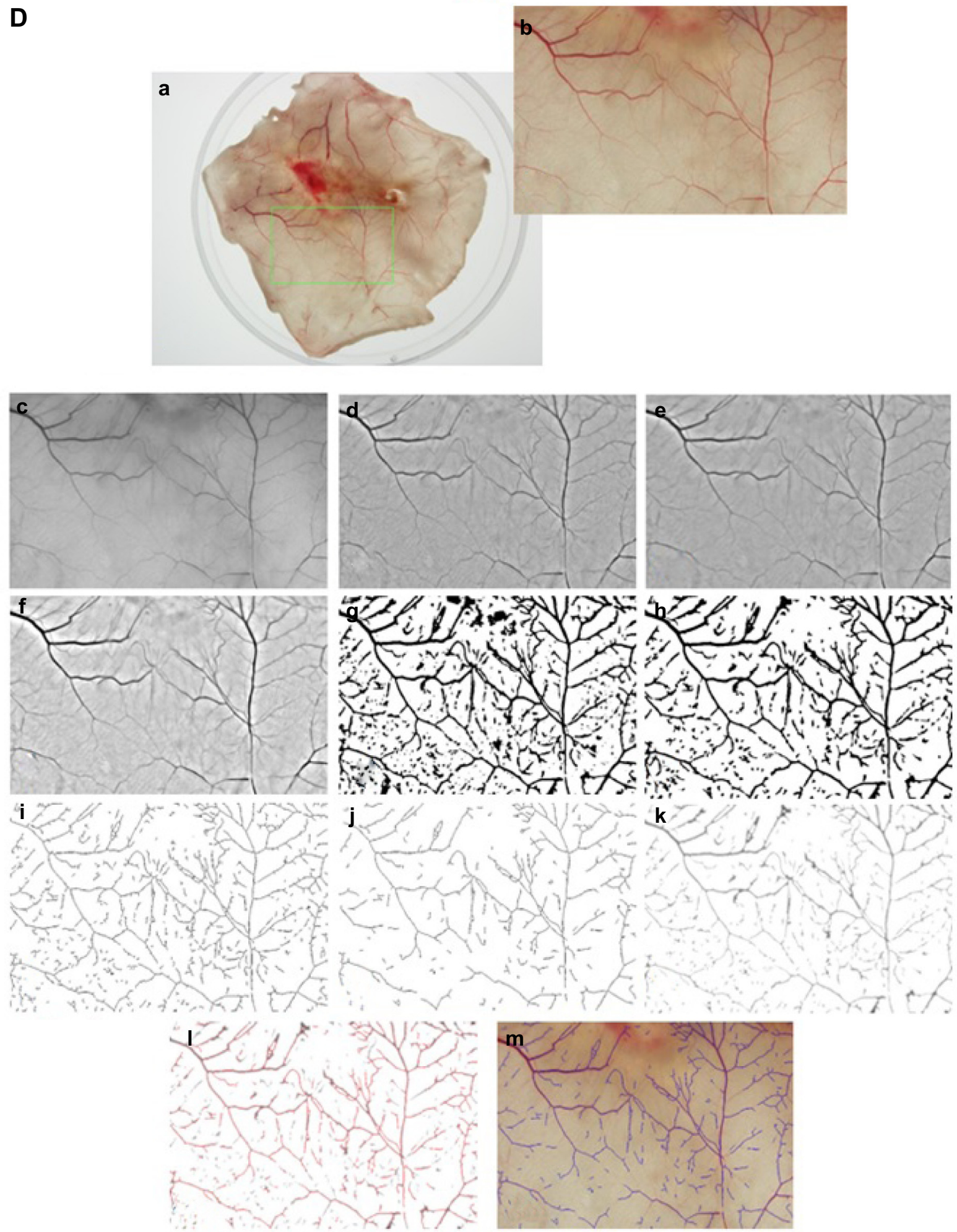

Figure 3 Quantitative image analysis of blood vessels.

Notes: Comparative vessel length over area $(\mathbf{A})$ and thickness measurements $(\mathbf{B})$ in rHuEPO-treated and untreated groups. Overall vessel thickness for treated rHuEPOtreated animals was $586.13 \mu \mathrm{m}$ and for untreated animals, $260.10 \mu \mathrm{m}$. Median value for rHuEPO-treated animals was $91.96 \mu \mathrm{m}$ and for untreated animals, $72.70 \mu \mathrm{m}$. Image processing pipeline went through the following steps (C): the target tissue (rHuEPO-treated) was extracted (a); selections of regions of interest (ROI) (b); converted to grayscale (c); high-pass filtering (d); anisotropic diffusion filtering (e); global contrast enhancement (f); binarization (g); unwanted segment removal (h); skeletonization (i); pruning (j); distance transformation (k); skeleton-over-distance transformation (l); skeleton-over-ROI (m). Image processing pipeline then went through the following steps (D): the target tissue (untreated rHuEPO) was extracted (a); selection of ROI (b); converted to grayscale (c); high-pass filtering (d); anisotropic diffusion filtering (e); global contrast enhancement (f); binarization (g); unwanted segment removal (h); skeletonization (i); pruning (j); distance transformation (k); skeleton-over-distance transformation (l); skeleton-over-ROI (m). EI and E2 are two sample specimens, taken from regenerated skins of rHUEPO treated animals for analysis of vessel length measurement and vessel thickness measurements. $\mathrm{Cl}$ and $\mathrm{C} 2$ are two sample specimens, taken from skins of untreated rHUEPO animals for analysis of vessel length measurement and vessel thickness measurements.

Abbreviations: rHuEPO, recombinant human erythropoietin; EPO, erythropoietin; w/o, without; EI, treated rHuEPO (day I4); E2 treated rHuEPO (day I4); CI, untreated rHuEPO (day 14); C2, untreated rHuEPO (day 14). 
percentage of re-epithelialized area as compared to the wound size.

\section{Histological analysis of skin regeneration}

The regeneration of the scald wound skin was further assessed by histological examinations, especially the analysis of neoangiogenesis, stimulation of cell proliferation, and stem cell recruitment in the skin injury sites by hematoxylin and eosin (H\&E) routine and other immunohistological staining. The quality of the regenerated skin was analyzed by histological observations of different parameters such as depth of the epidermal layer, epidermal stratification, the existence of papillary structures, and evidence of dermal innervation with blood vessels.

The frozen tissue sections of $14 \mu \mathrm{m}$ thickness were cut with a cryotome (model CM $3050 \mathrm{~S}$; Leica Microsystems) and stained with H\&E (Mayer's modification; Roth, Karlsruhe, Germany) using standard protocols. The stained samples were analyzed by light microscopy, and images were taken on an Olympus IX 51 microscope (Olympus Corporation) using a CC 12 camera and imaging software of the Olympus Cell family (Cell Imaging Software for Life Sciences Microscopy; Olympus Soft Imaging Systems GmbH, Münster, Germany).

For immunohistochemical or indirect immunoperoxidase staining, the thawed, air-dried, and fixed cryosections were blocked over a period of 10 minutes with $10 \%$ normal goat serum in phosphate-buffered saline (PBS; pH 7.4), and were subsequently incubated with an appropriate PBS dilution of primary monoclonal antibody for 1-2 hours at room temperature. Sections were then rinsed in PBS and incubated with a PBS solution containing 2\% secondary horseradish peroxidase conjugated goat anti-mouse antibody (DIANOVA GmbH, Hamburg, Germany) and 2\% normal rat serum for blocking. After incubation for 45 minutes to 1 hour at room temperature, binding of the secondary antibody was detected by incubation with 3-amino-9-ethylcarbazole (AEC) in sodium acetate buffer $(0.1 \mathrm{~mol} / \mathrm{L}, \mathrm{pH} 5.2)$ containing hydrogen peroxide. After rinsing in tap water, the sections were counterstained with hematoxylin Lillie's modification (ready-to-use formulation; DakoCytomation, Copenhagen, Denmark) and mounted in glycergel (Kaiser's glycerol gelatine; Merck KGaA, Darmstadt, Germany).

The cryosections for immunohistological staining were selected based on the HE staining results from different intervals (parts) of each specimen. The immunostaining results showed the proangiogenetic effect of EPO on tissue regeneration, particularly about epithelial covering, the presence of intact skin layers, and vascularization.
Hence, for the immunostaining we used the primary antibody against CD 71 as a proliferation marker for epithelial cells (CL071AP, 1:200; Cedarlane/Biozol, Eching, Germany); CD 31 as marker for new endothelial formation (MCA342R, 1:10,000; Bio-Rad Laboratories Inc., Hercules, CA, USA); CD 90 as a marker for mesenchymal stem cells (22211D, 1:200; BD Biosciences, San Jose, CA, USA); and nestin as neuronal progenitor and stem cell marker (MAB353, 1:400; EMD Millipore, Billerica, MA, USA).

\section{Statistical analysis}

The Student's $t$-test was used to compare samples using GraphPad software. $P<0.05$ was considered statistically significant.

\section{Results and discussion Vascularization rate in the mouse model}

The average wound vessel density on days 7 and 14 were $18.14 \%$ and $13.90 \%$, respectively, while vessel density was $6.77 \%$ and $8.07 \%$ on the same days, respectively, in the control group. Significant statistical difference was noted at both time points $(P<0.05)$ (Figure 4$)$. Galeano et $\mathrm{al}^{25}$ reported that $\mathrm{rHuEPO}$ increased scald wound re-epithelialization and reduced the time to final wound closure in mice; this result was obtained by giving injections of $\mathrm{rHuEPO}$ ( 400 units $/ \mathrm{kg} /$ day for 14 days) subcutaneously. In the present study, rHuEPO (352 U/ $\mathrm{kg} /$ day) is given in near-deep second-degree scald injury sites in a mouse model by infusion pump, in an attempt to mimic the in vivo circulation process; vascular blood vessel networks are also investigated in rHuEPO-treated and distilled watertreated mice. Furthermore, our results showed a significantly increased vascularization rate at days 7 and 14. However, a significant difference was observed after 14 days. The results

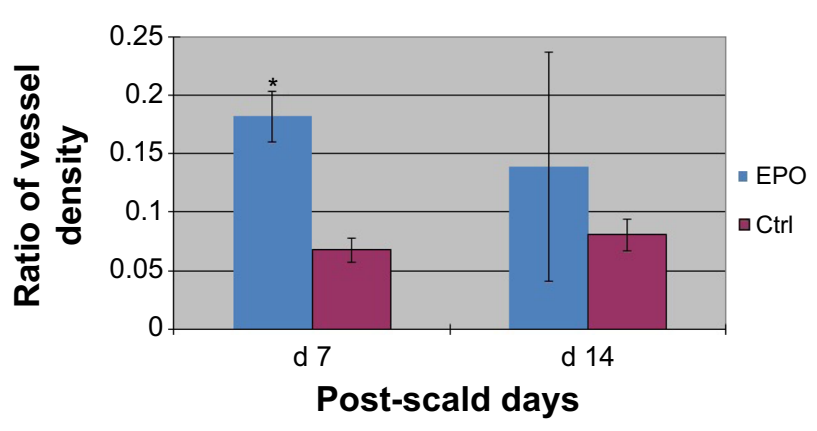

Figure 4 Difference in vessel density ratio.

Notes: The average wound vessel density on day 7 and day 14 was $18.14 \%$ and $13.90 \%$, respectively, while averages were $6.77 \%$ and $8.07 \%$, respectively, in the control group. Significant statistical difference at both time points was evident $(P<0.05)$. Values indicate the mean \pm SD of each group. $* P<0.05$ vs control group. Abbreviations: SD, standard deviation; Ctrl, control; vs, versus; d, day; EPO, erythropoietin. 
support the hypothesis that continuous application of $\mathrm{rHuEPO}$ in mice has a positive effect on the complex processes of wound healing. The detailed molecular signaling pathways remain unknown, but our results raise some hypotheses. For example, glycogen synthase kinase $3 b$ (GSK3 3$)$ is ubiquitously present in human cells, potentially to regulate the several physiological processes including ischemia injury. Recently, Wu et $\mathrm{al}^{30}$ investigated the local inhibition of GSK $3 \beta$ followed by elevated wnt signaling, which coordinates microtubule architecture and polarizes movement of skin stem cells toward wound sites for healing. Ohori et $\mathrm{al}^{31}$ reported that EPO protects cardiomyocytes from oxidant stress-induced apoptosis by the inhibitory phosphorylation of GSK $3 \beta$. Nishihara et $\mathrm{al}^{32}$ showed that EPO has potential to do additional roles for cardioprotection to preconditioned hearts by enhanced phosphorylation of GSK $3 \beta$. Therefore, it is more clinically relevant to apply GSK3 $\beta$ inhibitor agents like rHuEPO to organ injuries for protection as well as regeneration. Further research is needed to investigate the mechanisms involved. Toba et $\mathrm{al}^{33}$ reported the beneficial vasoprotective effects of rHuEPO, which included an improvement in endothelial dysfunction and the inhibition of vascular inflammation.

\section{Blood vessel segmentation and quantification}

Image-based quantitative assessment of angiogenesis in a mouse model revealed that the rHuEPO-treated group showed remarkable neovascularization and skin regeneration compared to the untreated rHuEPO group. First, a region of interest was selected from the original images and was converted into gray-scale for segmentation; the image was then inverted such that vessels appeared brighter than the background according to the conventions used by the vessel enhancement algorithms. Finally, segmentation was automatically performed by an algorithm-based program. Results showed that even the smallest vessels can be segmented using this optimized method. To evaluate the reliability of the segmentation, both images were compared, resulting in a significant difference between the original picture and the digital segmentation. High-pass methodology was also used in digital image processing for inhomogeneity correction of linear structure. Image processing algorithms were developed and compared with visual assessment of blood vessel networks in scalded mode. In the current study, we developed a semiautomated, quantitative technique for the quantitative assessment of blood vascularization in scald models. This technique provides a superior, easy-to-use, and quick method to detect the blood vessels in angiogenesis and neovascularization.
Neovascularization, the formation of new vessels, ${ }^{34}$ takes place during embryogenesis and in adults. ${ }^{35-37}$ Neovascularization is the formation of functional microvascular networks of blood vessels with red blood cell perfusion. Neovascularization differs from angiogenesis, which is a process for neoformation of blood vessels from pre-existing microvasculature. Both angiogenesis and neovascularization are key factors for promoting skin regeneration. Many experimental settings are excellent for evaluating larger arteries and veins, but these techniques are unable to detect microvasculature of smaller blood vessels between $100-500 \mu \mathrm{m} .^{24}$ Using image analysis methods, we were able to detect the microvascular networks of newly formed blood vessels (less than $500 \mu \mathrm{m}$ ) that participate in the healing process, and provide not only nutrition and oxygen to growing tissues but also provide necessary growth factors to growing tissue cells for complete skin regeneration.

Further, concerning the blood vessel's thickness, we found significant differences between the infusion $\mathrm{rHuEPO}$ pumping mouse group and the untreated mouse group. The rHuEPO-treated animals exhibited a typical array of blood vessels with diameters of around $75 \mu \mathrm{m}$.

\section{Skin regeneration by rHuEPO gel in the rat scald injury model}

The scald wound injury was well tolerated by the animals. No animals died during the procedure or postoperatively, and no signs of infection were found at the injury sites. After local application of rHuEPO gel to intradermal wounds and after tangential excision with a dermatome $(n=8)$, an average of $91 \%$ of the wound area was clinically re-epithelialized compared to $85 \%$ re-epithelialization in the control group $(n=8)$, which was statistically not significant $(P=0.344)$. Nonetheless, from the clinical aspect, the wounds treated with $\mathrm{rHuEPO}$ showed a better tendency to heal. At day 7, re-epithelialization levels of skin defects after tangential excision increased to an average of $98.8 \%$ in the rHuEPO-treated group compared to an $88 \%$ average in the controls, which was statistically significant $(P=0.031$; Figure 5A). Our results show that the use of locally applied rHuEPO is a rational way to improve re-epithelialization in scald wounds, suggesting a key role for $\mathrm{rHuEPO}$ in wound healing after local application (Figure 6).

\section{Histological analysis of skin regeneration}

The event of wound healing was first assessed macromorphologically after sacrifice, and was subsequently assessed microscopically by intensive histological studies. The parameters of tissue regeneration, re-epithelialization, 


\section{A \\ Re-epithelialization}
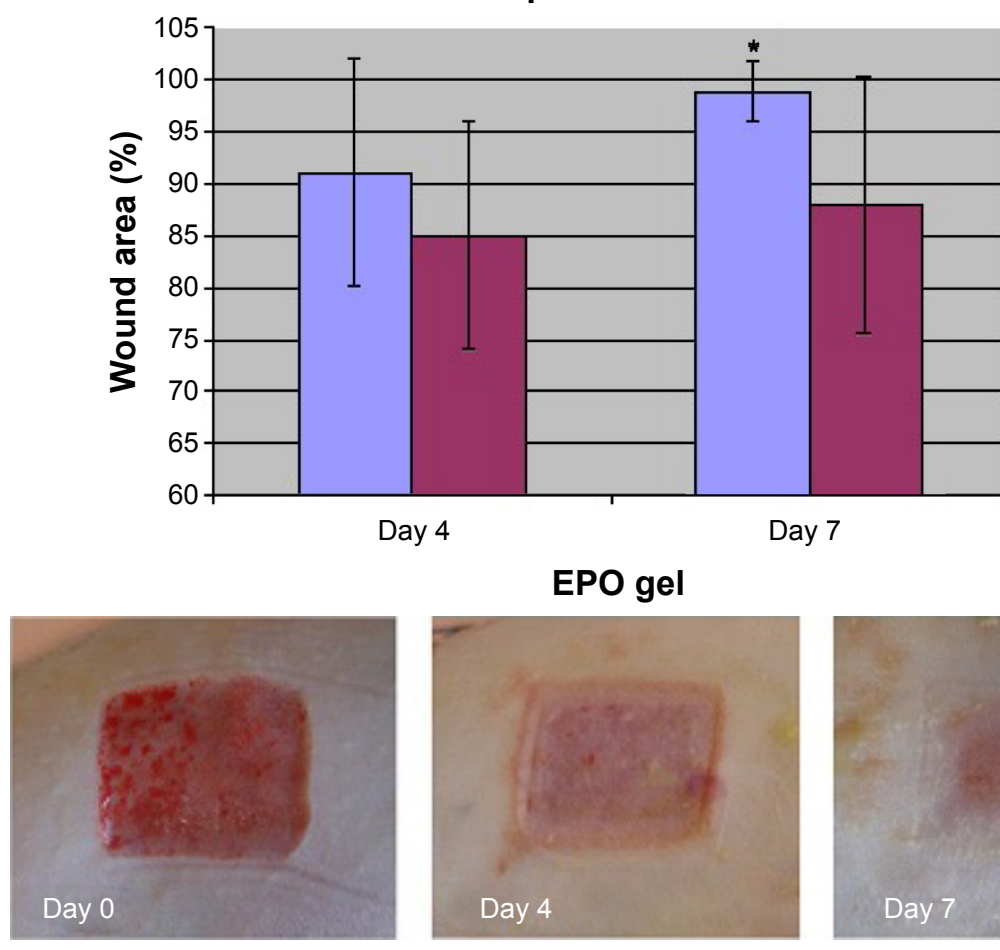

- Control gel

B

Day 4

Day 7

EPO gel

C

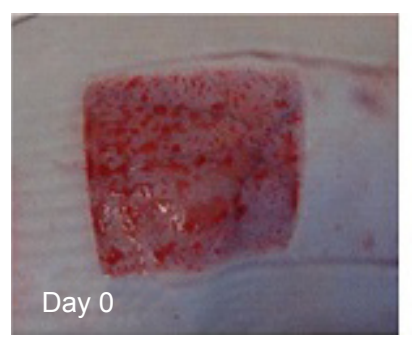

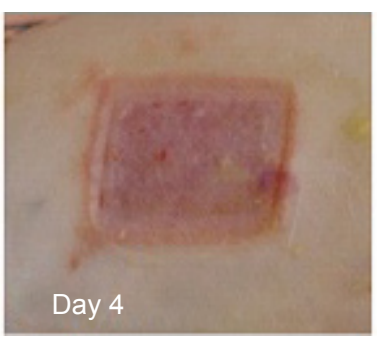

Control



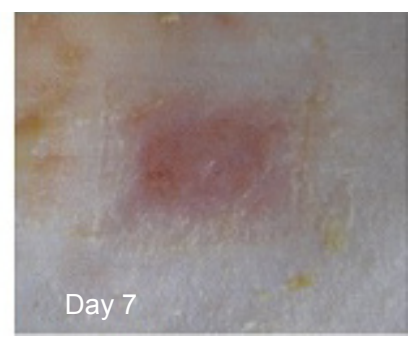

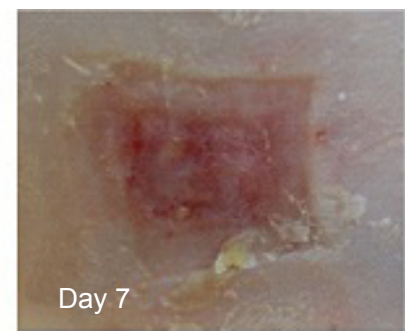

Figure 5 Re-epithelialization of skin in rHuEPO-treated and untreated groups.

Notes: Re-epithelialization level at day 4 and day $7(\mathbf{A})$ : histological scoring of the results of scald regeneration in rats treated with rHuEPO or vehicle at different healing times (4 and 7 days). Representative photomacroscopic images of wounds directly after tangential excision (day 0 ), and on day 4 and 7 after wounding with (B) EPO gel treated and $(\mathbf{C})$ control (no EPO gel). In the rHuEPO group, we noticed a significant decrease in wound size and quicker wound closure than in the control group at day 7. In addition, we found increased epithelial covering in the rHuEPO treatment group than in the control group. A considerably better skin regeneration, re-epithelialisation, and wound closure, and visibly less scar formation was found on day 4 in rats with topical rHuEPO treatment as compared to controls. The final score for epithelialization of skin regeneration for each animal specimen is demonstrated as the sum of the three partial score values. Values are the mean \pm standard error of the mean of each group. $* P<0.05$ versus control group.

Abbreviations: EPO, erythropoietin; rHuEPO, recombinant human erythropoietin.

wound closure, and especially scar healing effects were thereby evaluated and compared between rHuEPO-treated and control groups.

\section{Microscopic assessment of wound healing}

The histological evaluation of immunostaining by visual detection of skin regeneration markers such as CD31, CD 90, CD 71, and nestin indicated a visible improvement of scald wound healing with respect to epithelial covering, neovascularization, proliferation, and mobilization of stem cells in rHuEPO-treated animals in comparison with animals in the experimental control group.
In detail, in the group with rHuEPO-gel application (rat numbers 21,24$),{ }^{4}$ we detected at day 7 increased tissue regeneration, demonstrated by improved re-epithelialization. We also noted the accelerated formation of a new, thicker covering of epithelia and more intact skin layers, and increased neovascularization. Increased neovascularization was associated with more innervation in blood vessels (CD 31), increased proliferation (CD 71), and mobilization of stem cells (CD 90, nestin) when compared with animals of the experimental control group (rat numbers 22, 23) (Figure 7).

Webb et $a l^{38}$ showed that actively cycling epidermal cells exhibited high levels of expression of CD 71 
A

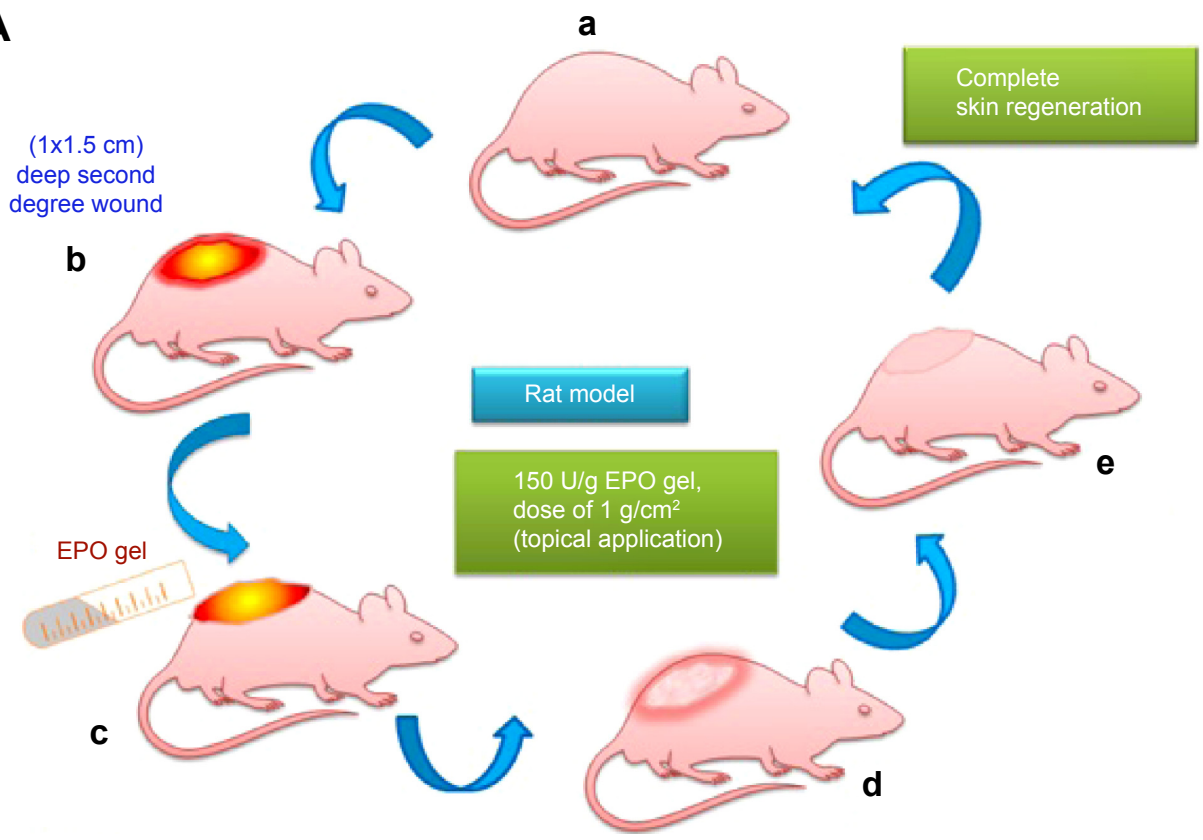

B

a

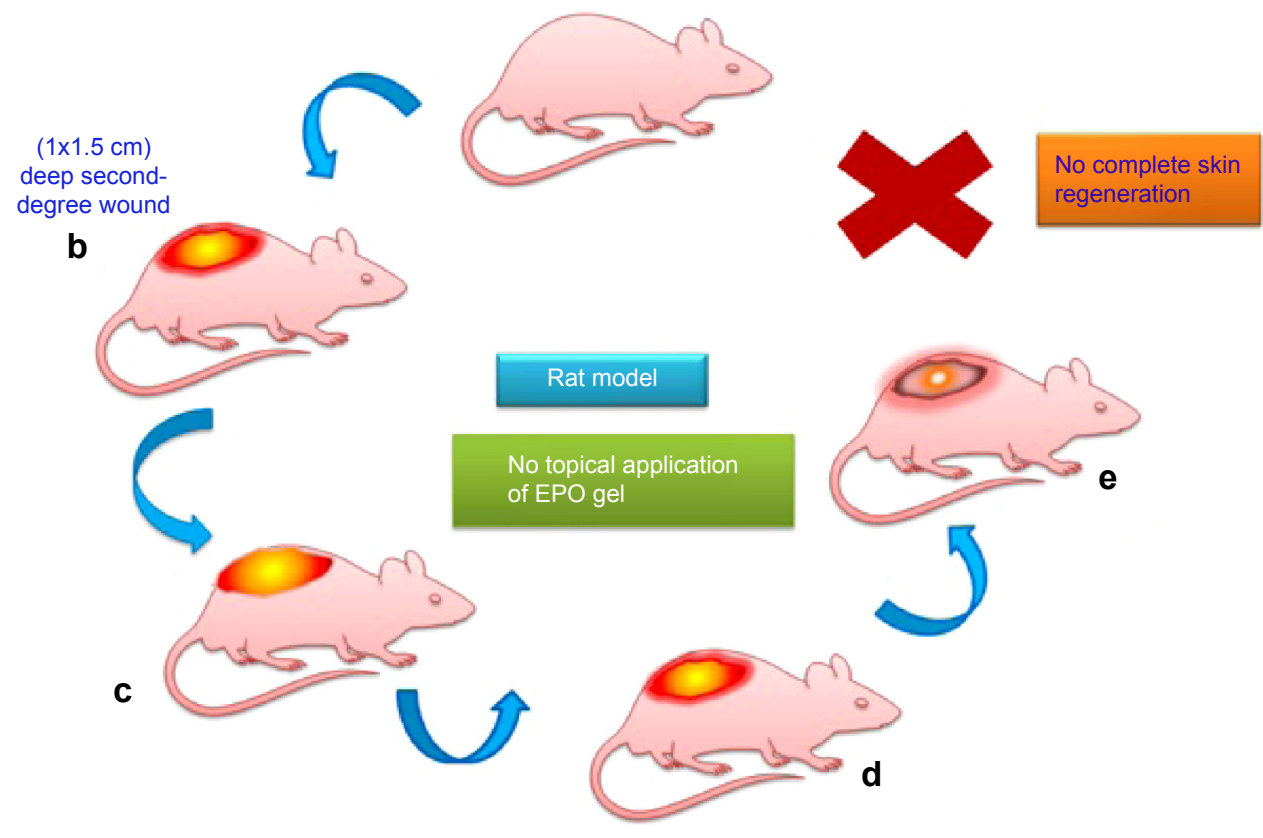

Figure 6 Diagrammatic representation of EPO treated and untreated rat model for skin regeneration.

Notes: Model of tangential excision in 6-8-week-old female rats weighing 200-250 g EPO treated (A, a-e). Each animal received four dorsolateral skin defects (I $\times 1.5 \mathrm{~cm})$ via tangential excision of $0.2 \mathrm{~mm}$ using a dermatome (Aesculap Inc., Center Valley, PA, USA). Every second day the rats were treated with rHuEPO hydrogel over the dermal wound bed ( $150 \mathrm{IU} \mathrm{rHuEPO} / \mathrm{g}$, dose of $\left.\mathrm{I} \mathrm{g} / \mathrm{cm}^{2}\right)$. Model of tangential excision in 6-8-week-old female rats weighing 200-250 g untreated with EPO (B, a-e). Each animal received four dorsolateral skin defects $(I \times I .5 \mathrm{~cm})$ via tangential excision of $0.2 \mathrm{~mm}$. These rat models are controls (no topical application of rHuEPO).

Abbreviations: EPO, erythropoietin; rHuEPO, recombinant human erythropoietin.

proliferation-related surface marker, while quiescent stem cells showed extremely low levels of CD 71. CD 71 is expressed at higher levels on cells exhibiting a high proliferation rate and is upregulated in lymphocytes during proliferative responses to antigens or mitogens. CD 71 is not expressed on resting lymphocytes. A part of the skin generation niche, CD 71 is expressed on marrow stromal cells of bone marrow. ${ }^{39} \mathrm{CD} 71$ is also expressed by activated $\mathrm{T}$ and $\mathrm{B}$ lymphocytes, ${ }^{40}$ macrophages, and all proliferating cells. We found significantly higher expression of CD 71 in $\mathrm{rHuEPO}-$ treated areas than in untreated areas in rats.

CD 31 acts as a regulatory molecule in vascular biology because it is highly expressed on endothelial and blood cells. Walls of blood vessels are lined by an exceedingly thin layer 
A

a

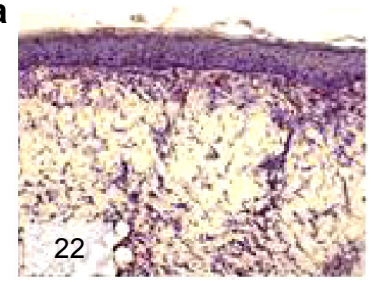

b

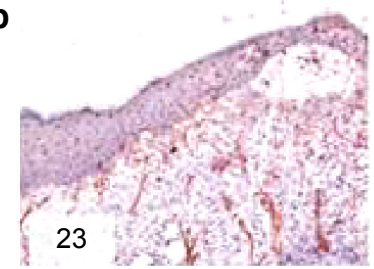

C



d

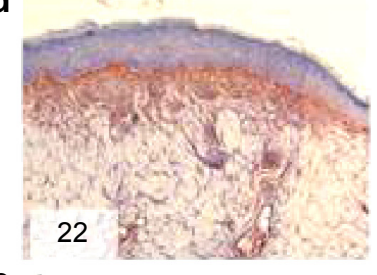

e

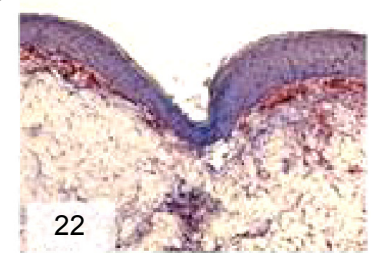

f

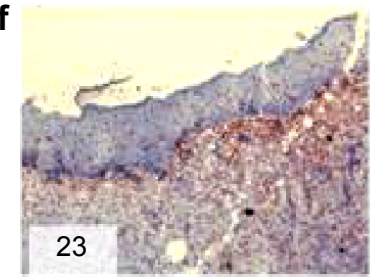

Control gel
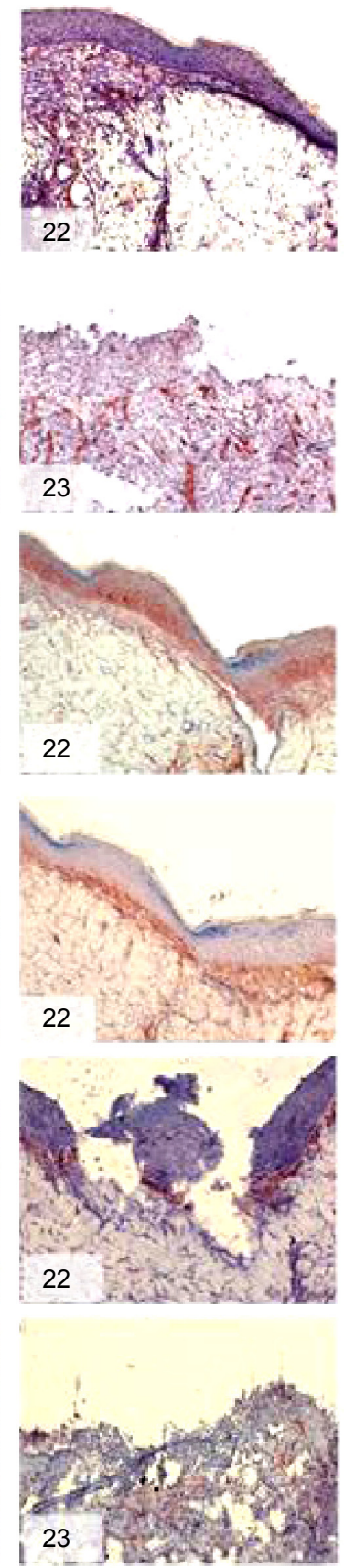

B



EPO gel



Figure 7 Representative immunostaining of scalded skin tissue specimens from rats on day 7 after wounding and topical application of rHuEPO gel or its vehicle (control gel). Notes: (A) control group without rHuEPO (vehicle only), rat number 22 (a, c, d, e), rat number 23 (b, f); (B) rHuEPO group, rat number 21 (a, c, d, e), rat number 24 (b, f). Staining to assess CD 3I (a, b); CD 7I (c); CD 90 (d); nestin (e, f). Arrows indicate the location of expression of these different markers. Magnification I00×, scale bar is $200 \mu \mathrm{m}$. Abbreviations: EPO, erythropoietin; rHuEPO, recombinant human erythropoietin.

of endothelial cells. Staining of blood vessels with CD 31 antibodies has been shown to be suitable for the identification of angiogenesis. ${ }^{41}$ It was observed in the present study that vascular networks formed early within the rHuEPO-treated area on day 7 , as evidenced by positive CD 31 staining to visualize regenerating structures in the dermis and epidermis. We found significantly higher levels of vascular endothelial markers (CD 31) in rHuEPO-treated areas than in untreated rats, suggesting that $\mathrm{CD} 31$-positive cells may contribute to newly formed blood vessels.

Nakamura et $\mathrm{al}^{42}$ detected CD 90-positive cells in cultured normal human epidermal keratinocytes and adult skin. They showed that the basal layer of the normal human epidermis expressed CD $90 .{ }^{42}$ Stem cells isolated from 
the dermal sheath of hair follicles also express CD $90 .{ }^{43}$ Although it has been a hypothesis that CD 90-positive fibroblasts are dynamically involved in wound healing, ${ }^{44,45}$ Mercati et $\mathrm{al}^{46}$ analyzed this hypothesis using immunohistochemical methods and found rich positivity to CD 90 protein in the dermal cells located in the lower part of the anagen hair follicle. In the present study, we immunohistochemically evidenced a clear and abundant positivity to CD 90 protein in the rHuEPO-treated area in the scalded mouse model.

The fibroblast cells within granulation tissue play a central role in wound contraction, but the interaction between fibroblasts and the surrounding extracellular matrix remains unclear. In normal wounds, fibroblasts can become scars, but it is possible to have re-epithelialization of skin occur without scarring through the influence of rHuEPO. We hypothesized that rHuEPO fulfills a master role in scarless healing. In the present study, we tested $\mathrm{rHuEPO}$ in comparison with a control group to evaluate rHuEPO gel and infusion pumping efficacy at the wound site for skin regeneration.

Nestin-expressing pluripotent stem cells were found in the bulge area of hair follicles. ${ }^{47,48}$ Further, it was also reported that nestin-expressing stem cells migrated from the bulge area toward wounded skin, ${ }^{49}$ where these nestinpositive cells could participate in wound healing processes for new hair follicle generation and skin regeneration. ${ }^{45}$ In the current study, we found significantly higher nestinexpressing stem cells in rHuEPO-treated than in untreated small animals, suggesting that nestin-positive cells may contribute to complete skin regeneration. The results of this study lead to the assumption of much more potential efficacy of topical rHuEPO gel in accelerating skin regeneration following scald injuries.

\section{Conclusion}

The present study concluded that complete skin regeneration of deep second-degree scalding injuries is possible either by infusion pumping of rHuEPO or by topical application of rHuEPO gel. It was shown that rHuEPO administration accelerated neovascularization, re-epithelization, and wound healing. Infusion pumping of rHuEPO, an attempt to mimic the in vivo circulation process, and topical application of $\mathrm{rHuEPO}$ gel allowed complete skin regeneration. Numbers of new circulatory blood vessel networks were significantly higher in the rHuEPO pumping group than in the untreated group. Using image analysis methods, we were able to detect the microvascular networks of newly formed blood vessels (less than $500 \mu \mathrm{m})$. These blood vessels participate in the healing process, providing not only nutrition and oxygen to grow tissues but also necessary growth factors to grow tissue cells for complete skin regeneration. Higher expression of stem cell markers such as CD 31, CD 90, CD 71, and nestin were observed in rHuEPO gel-treated rats than in untreated rats. These markers actively contribute to the wound-healing process, particularly in the generation of new hair follicles and skin. Further, enhanced re-epithelialization was found in $\mathrm{rHuEPO}$ gel-treated rats as compared to untreated rats. This finding has important clinical implications with regard to the potential use of $\mathrm{rHuEPO}$ gel at the injury site in scald patients.

\section{Disclosure}

The authors report no conflicts of interest in this work.

\section{References}

1. World Health Organization [webpage on the Internet]. WHO Fact Sheet No 310: The top ten causes of death. Geneva: World Health Organization; 2004 [last updated May 2014]. Available from: www.who.int/ mediacentre/factsheets/fs310/en. Accessed June 8, 2010.

2. World Health Organization [webpage on the Internet]. The global burden of disease: 2004 update. Geneva: World Health Organization; 2008.

3. Sánchez JL, Perepérez SB, Bastida JL, Martínez MM. Cost-utility analysis applied to the treatment of scald patients in a specialized center. Arch Surg. 2007;142(1):50-57; discussion 57.

4. World Burn Foundation [homepage on the Internet]. Available from http:// www.burnfoundation.com/e/index.htm. Accessed March 23, 2015.

5. Marshall SW, Runyan CW, Bangdiwala SI, Linzer MA, Sacks JJ, Butts JD. Fatal residential fires: who dies and who survives? JAMA. 1998;279(20):1633-1637.

6. Alden NE, Bessey PQ, Rabbitts A, Hyden PJ, Yurt RW. Tap water scalds among seniors and the elderly: socio-economics and implications for prevention. Scalds. 2007;33(5):666-669.

7. Centers for Disease Control [webpage on the Internet]. Web-based injury and statistics query and reporting system (WISQARS ${ }^{\mathrm{TM}}$ ) 2009 of Prevention of fire and scald injuries. Atlanta, GA: Centers for Disease Control; 2010. Available from: webappa.cdc.gov/sasweb/ncipc/mortrate9.html. Accessed April 2, 2010.

8. Avni T, Levcovich A, Ad-El DD, Leibovici L, Paul M. Prophylactic antibiotics for scalds patients: systematic review and meta-analysis. BMJ. 2010;340:c241.

9. Bader A, Lorenz K, Richter A, et al. Interactive role of trauma cytokines and erythropoietin and their therapeutic potential for acute and chronic wounds. Rejuvenation Res. 2011;14(1):57-66.

10. Elliott MJ, De Coppi P, Speggiorin S, et al. Stem-cell-based, tissue engineered tracheal replacement in a child: a 2-year follow-up study. Lancet. 2012;380(9846):994-1000.

11. Galeano M, Altavilla D, Cucinotta D, et al. Recombinant human erythropoietin stimulates angiogenesis and wound healing in the genetically diabetic mouse. Diabetes. 2004;53(9):2509-2517.

12. Sorg H, Krueger C, Schulz T, Menger MD, Schmitz F, Vollmar B. Effects of erythropoietin in skin wound healing are dose related. FASEB J. 2009;23(9):3049-3058.

13. Bader A, Ebert S, Giri S, et al. Skin regeneration with conical and hair follicle structure of deep second-degree scalding injuries via combined expression of the EPO receptor and beta common receptor by local subcutaneous injection of nanosized rhEPO. In Nanomedicine. 2012;7: 1227-1237.

14. Hamed S, Ullmann Y, Masoud M, Hellou E, Khamaysi Z, Teot L. Topical erythropoietin promotes wound repair in diabetic rats. J Invest Dermatol. 2010;130(1):287-294. 
15. Martin P. Wound healing - aiming for perfect skin regeneration. Science. 1997;276(5309):75-81.

16. Haroon ZA: Angiogenesis and oxygen transport in solid tumors. In: Teicher BA, editor. Antiangiogenic Agents in Cancer Therapy. Totowa, NJ: Humana Press Inc; 1999:3-21.

17. Tredget EE. The basis of fibrosis and wound healing disorders following thermal injury. J Trauma. 2007;62(6 Suppl):S69.

18. Folkman J, Shing Y. Angiogenesis. J Bio Chem. 1992;267:10931-10934.

19. Carlini RG, Reyes AA, Rothstein M. Recombinant human erythropoietin stimulates angiogenesis in vitro. Kidney Int. 1995;47(3):740-745.

20. Ribatti D, Presta M, Vacca A, et al. Human erythropoietin induces a pro-angiogenic phenotype in cultured endothelial cells and stimulates neovascularization in vivo. Blood. 1999;93(8):2627-2636.

21. Kawachi K, Iso Y, Sato T, et al. Effects of erythropoietin on angiogenesis after myocardial infarction in porcine. Heart Vessels. 2012; 27(1):79-88.

22. McDonald DM, Choyke PL. Imaging of angiogenesis: from microscope to clinic. Nat Med. 2003;9(6):713-725.

23. Machens HG, Grzybowski S, Bucsky B, et al. A technique to detect and to quantify fasciocutaneous blood vessels in small laboratory animals ex vivo. J Surg Res. 2006;131(1):91-96.

24. McDonald DM, Choyke PL. Imaging of angiogenesis: from microscope to clinic. Nat Med. 2003;9(6):713-725.

25. Galeano M, Altavilla D, Bitto A, et al. Recombinant human erythropoietin improves angiogenesis and wound healing in experimental scald wounds. Crit Care Med. 2006;34(4):1139-1146.

26. Gonzalez RC, Woods RE. Digital Image Processing. 2nd ed. Upper Saddle River, NJ: Prentice-Hall; 2002

27. Weickert J. Anisotropic Diffusion in Image Processing. ECMI Series. Stuttgart, Germany: Teubner Verlag; 1998.

28. Coeurjolly D, Montanvert A. Optimal separable algorithms to compute the reverse euclidean distance transformation and discrete medial axis in arbitrary dimension. IEEE Trans Pattern Anal Mach Intell. 2007;29(3): 437-448.

29. Saito T, Toriwaki JO. New algorithms for euclidean distance transformation on an n-dimensional digitized picture with applications. Pattern Recogn. 1994;27:1551-1565.

30. Wu X, Shen QT, Oristian DS, et al. Skin stem cells orchestrate directional migration by regulating microtubule-ACF7 connections through GSK3beta. Cell. 2011;144(3):341-352.

31. Ohori K, Miura T, Tanno M, et al. Ser9 phosphorylation of mitochondrial GSK-3beta is a primary mechanism of cardiomyocyte protection by erythropoietin against oxidant-induced apoptosis. Am J Physiol Heart Circ Physiol. 2008;295(5):H2079-H2086.

32. Nishihara M, Miura T, Miki T, et al. Erythropoietin affords additional cardioprotection to preconditioned hearts by enhanced phosphorylation of glycogen synthase kinase-3 beta. Am J Physiol Heart Circ Physiol. 2006;291(2):H748-H755.

33. Toba H, Morishita M, Tojo C, et al. Recombinant human erythropoietin ameliorated endothelial dysfunction and macrophage infiltration by increasing nitric oxide in hypertensive 5/6 nephrectomized rat aorta Eur J Pharmacol. 2011;656(1-3):81-87.
34. Risau W, Flamme I. Vasculogenesis. Annu Rev Cell Dev Biol. 1995;11: 73-91.

35. Asahara T, Murohara T, Sullivan A, et al. Isolation of putative progenitor endothelial cells for angiogenesis. Science. 1997;275(5302):964-967.

36. Asahara T, Masuda H, Takahashi T, et al. Bone marrow origin of endothelial progenitor cells responsible for postnatal vasculogenesis in physiological and pathological neovascularization. Circ Res. 1999; 85(3):221-228.

37. Urbich C, Dimmeler S. Endothelial progenitor cells: characterization and role in vascular biology. Circ Res. 2004;95(4):343-353.

38. Webb A, Li A, Kaur P. Location and phenotype of human adult keratinocyte stem cells of the skin. Differentiation. 2004;72(8):387-395.

39. Pittenger MF, Mackay AM, Beck SC, et al. Multilineage potential of adult human mesenchymal stem cells. Science. 1999;284(5411):143-147.

40. Salmon M, Bacon, PA, Symmons DP, Walton KW. Transferrin receptor expression by stimulated cells in mixed lymphocyte culture. Immunology. 1985;54(3):559-564.

41. Schmidt D, von Hochstetter AR. The use of CD31 and collagen IV as vascular markers. A study of 56 vascular lesions. Pathol Res Pract. 1995;191(5):410-414.

42. Nakamura Y, Muguruma Y, Yahata T, et al. Expression of CD90 on keratinocyte stem/progenitor cells. Br J Dermatol. 2006;154(6): $1062-1070$

43. Hoogduijn MJ, Gorjup E, Genever PG. Comparative characterization of hair follicle dermal stem cells and bone marrow mesenchymal stem cells. Stem Cells Dev. 2006;15(1):49-60.

44. Koumas L, King AE, Critchley HO, Kelly RW, Phipps RP. Fibroblast heterogeneity: existence of functionally distinct Thy $1(+)$ and Thy 1(-) human female reproductive tract fibroblasts. Am J Pathol. 2001;159(3):925-935.

45. Koumas L, Smith TJ, Feldon S, Blumberg N, Phipps RP. Thy-1 expression in human fibroblast subsets defines myofibroblastic or lipofibroblastic phenotypes. Am J Pathol. 2003;163(4):1291-1300.

46. Mercati F, Pascucci L, Ceccarelli P, Dall'Aglio C, Pedini V, Gargiulo AM. Expression of mesenchymal stem cell marker CD90 on dermal sheath cells of the anagen hair follicle in canine species. Eur J Histochem. 2009; 53(3):159-166.

47. Liu F, Uchugonova A, Kimura H, et al. The bulge area is the major hair follicle source of nestin-expressing pluripotent stem cells which can repair the spinal cord compared to the dermal papilla. Cell Cycle. 2011;10(5):830-839.

48. Uchugonova A, Duong J, Zhang N, Konig K, Hoffman RM. The bulge area is the origin of nestin-expressing pluripotent stem cells of the hair follicle. J Cell Biochem. 2011;112(8):2046-2050.

49. Amoh Y, Maejima H, Niiyama S, et al. Hair follicle stem cell marker nestin expression in regenerating hair follicles of patients with alopecia areata. Eur J Dermatol. 2011;21(2):209-212.

\section{Publish your work in this journal}

Drug Design, Development and Therapy is an international, peerreviewed open-access journal that spans the spectrum of drug design and development through to clinical applications. Clinical outcomes, patient safety, and programs for the development and effective, safe, and sustained use of medicines are a feature of the journal, which

\section{Dovepress}

has also been accepted for indexing on PubMed Central. The manuscript management system is completely online and includes a very quick and fair peer-review system, which is all easy to use. Visit http://www.dovepress.com/testimonials.php to read real quotes from published authors. 